\title{
Summary of the IADR Cariology Research Group Symposium, Barcelona, Spain, July 2010: New Directions in Cariology Research
}

\author{
A.R. Vieira ${ }^{a}$ A. Modesto ${ }^{a} \quad$ A. Ismail ${ }^{b} \quad$ R. Watt ${ }^{c}$ \\ a University of Pittsburgh School of Dental Medicine, Pittsburgh, Pa., and b Temple University, Maurice H. Kornberg \\ School of Dentistry, Philadelphia, Pa., USA; ' University College London Medical School, London, UK
}

\section{Key Words}

IADR Cariology Research Group Symposium • Etiology •

Genetic studies · Population-based cohort techniques

\begin{abstract}
Caries remains the most prevalent noncontagious biofilmmediated disease in humans. It is clear that the current approaches to decrease the prevalence of caries in human populations, including water fluoridation and school-based programs, are not enough to protect everyone. The scientific community has suggested the need for innovative work in a number of areas in cariology, encompassing disease etiology, epidemiology, definition, prevention, and treatment. In this symposium, two of these areas, dealing specifically with etiological aspects of caries were discussed: (1) systematic research on caries risk assessment using population-based cohort techniques, and (2) genetic studies to identify genes and genetic markers of diagnostic, prognostic, and therapeutic value. This paper summarizes these presentations.
\end{abstract}

Copyright $\odot 2012$ S. Karger AG, Basel
(C) 2012 S. Karger AG, Basel

0008-6568/12/0464-0346\$38.00/0

Fax +4161306 1234

E-Mail karger@karger.ch

www.karger.com
Accessible online at: www.karger.com/cre
Although it has been more than 100 years since Miller first described the etiopathogenesis of caries, this condition remains the most prevalent noncontagious biofilmmediated disease in humans. It is clear that the current approaches to decrease the prevalence of caries in human populations are not enough to protect everyone. While water fluoridation is not a universal strategy, there is no evidence that the school-based programs are responsible for the decline in caries rates seen. The worldwide caries decline has been attributed to the widespread use of fluorides, which were enforced by the addition of fluoride to the toothpaste. The NIH Consensus Development Program released a statement in 2001 and listed six major clinical caries research directions. Two of these directions deal specifically with etiological aspects of caries: (1) systematic research on caries risk assessment using population-based cohort techniques, and (2) genetic studies to identify genes and genetic markers of diagnostic, prognostic, and therapeutic value. This symposium provided a unique insight of how these two areas of caries research have been approached. Although aspects related to the influences of dental biofilm on caries are also a great area of interest, they were not the primary focus of the symposium. 


\section{Evidence-Based Risk Assessment for Caries (Presenter A. Ismail)}

Evidence-based dentistry provides an approach of systematically collecting and analyzing scientific evidence relating to the patient's oral and medical conditions and history, through the dentist's clinical expertise and the patient's treatment needs and preferences [Ismail and Bader, 2004]. Although the concept appears fundamentally simple and reasonable, clinicians encounter barriers in their attempts to incorporate evidence-based dentistry into clinical practice [Kao, 2006]. The significant difference in the evidence-based care, in contrast with relying on techniques, materials and treatments learned years earlier, is the emphasis on clinical decision-making based on the body of evidence present in the scientific literature. Expert opinions and information based on case studies are not considered to be sufficiently strong or credible in the decision-making process [Ismail and Bader, 2004]. The process should be based on integrating the scientific basis for clinical care using thorough, unbiased reviews and the best available scientific evidence at any one time, with clinical and patient factors to make the best possible decision(s) about appropriate care for specific clinical circumstances for the individual patient. Best evidence refers to information obtained from randomized controlled clinical trials, nonrandomized controlled clinical trials, cohort studies, case-control studies, crossover studies, cross-sectional studies, case studies or, in the absence of scientific evidence, the consensus opinion of experts in the appropriate fields of research or clinical practice [ADA Policy on Evidence-Based Dentistry, 2008].

Risk factors are characteristics, conditions, or behaviors that increase the possibility of disease and can be environmental, behavioral, or biological factors confirmed by temporal sequence usually in longitudinal studies, which if present, directly increase the probability of a disease occurring, and if absent or removed reduce the probability of a disease occurring. Risk factors are part of the causal chain, or expose the host to the causal chain. Once disease occurs, removal of a risk factor may not result in a cure, but may reduce the future episodes of disease [Beck, 1998]. A person's caries risk can vary with time since many risk factors are changeable [Selwitz et al., 2007]. Some risk factors or mediators/moderators in the causal pathway are socioeconomic position, cultural norms (dietary norms), religiosity, social support, resiliency, unemployment, and housing (lead paint, drinking water, crime, family support).

Summary of the IADR Cariology

Research Group Symposium
There is a wide variation of definition, criteria and analysis of dental caries. In general the studies are based in staging of the disease process considering cavitation or total caries experience. Dental caries is initially reversible and may be halted at any stage, even when some dentin or enamel is destroyed, provided that enough biofilm can be removed, the site remains biofilm-free and exposed to the oral environment. It is a chronic disease that progresses slowly in most people [Selwitz et al., 2007].

Diagnostic threshold is a term that describes the cutoff level used in an arbitrary decision of what to classify as diseased and what to classify as sound. This can be represented in the form of an iceberg. The peak of the iceberg represents gross or frank dentin caries (the so-called $\mathrm{D}_{4}$ and more limited $\mathrm{D}_{3}$ caries lesions) that rests on increasingly larger volumes of less extensive decay at the $\mathrm{D}_{2}$ (enamel cavity) and more limited $\mathrm{D}_{1}$ (white- or brownspot caries lesions) levels of severity [Pitts, 2004].

The opportunities presented by a shift in thinking about caries cannot be fully embraced without first changing the manner in which caries is talked about, viewed, and approached in terms of treatment. Whereas in the past caries was addressed with a drill-and-fill approach, the emphasis today is to remineralize, rejuvenate, and replace tooth structure. Further, an emphasis on prevention focuses efforts on early caries detection, diagnosis, assessment, and management.

'Systems thinking' has been defined as an approach to problem solving by viewing problems as parts of an overall system, rather than reacting to a specific part, outcomes or events and potentially contributing to further development of unintended consequences ${ }^{1}$. The latest flagship report from the Alliance for Health Policy and Systems Research offers a practical approach to strengthening health systems through 'systems thinking' showing how to better capture the wisdom of diverse health system stakeholders in designing solutions to system problems. It also suggests ways to more realistically forecast how health systems might respond to systemstrengthening interventions, while also exploring potential synergies and dangers among those interventions [de Savigny and Adam, 2009].

\footnotetext{
These thoughts are derived from the General Systems Theory of Von Bertalanffy [1968]. This author developed the idea that organized wholes of any kind should be describable, and to a certain extent explainable by means of the same categories, and ultimately by one and the same formal apparatus. His General Systems Theory triggered a whole movement, which has tried to identify invariant structures and mechanisms across different kinds of organized wholes. This approach has been applied to organizational, societal, and ecological issues [Schwaninger, 2003/4].
}

Caries Res 2012;46:346-352 
The profession today understands the caries process as an interactive, dynamic chemical/bacterial process that occurs between the complex biofilm, tooth structures and saliva system. Caries does not equate to cavities, and it should not be managed only with materials. Further, cavitation may occur at different stages/surfaces of tooth structure, and the process might not be visible. It is best to prevent cavities at the earliest signs of demineralization, noting that it could be prevented if clinicians are trained to diagnose and manage the disease.

Takahashi and Nyvad [2008] proposed an extension of the caries ecological hypothesis to explain the relation between dynamic changes in the phenotypic/genotypic properties of plaque bacteria and the demineralization/ remineralization balance of the caries process. From an ecological point of view it is therefore not only important to describe which bacteria are involved in caries, but also to know what the bacteria are doing. In this hypothesis, dental plaque is a dynamic microbial ecosystem in which non-mutans bacteria are the key players for maintaining dynamic stability. However, when sugar is supplied frequently or salivary secretion is too scarce to neutralize or clear the acids produced, the $\mathrm{pH}$ decreases in the plaque may enhance the acidogenicity and aciduricity of the non-mutans bacteria adaptively. Under such conditions the population of the 'low $\mathrm{pH}$ ' non-mutans streptococci and Actinomyces then increases via acid selection, leading to a microbial shift to a more acidogenic microflora. These changes in the phenotype of the microflora may shift the demineralization/remineralization balance from 'net mineral gain' to 'net mineral loss' and initiate lesion development (acidogenic stage). At this stage, lesion development could also be arrested with de-adaptation of the microflora, provided that the mineral balance is restored to a 'net mineral gain' by reduced environmental acidification. If prolonged acidic environments prevail, lesion development ('net mineral loss') is likely to progress. In these environments, more aciduric bacteria such as mutans streptococci, bifidobacteria and lactobacilli may replace the 'low-pH' non-mutans bacteria and further accelerate the caries process (aciduric stage) [Takahashi and Nyvad, 2008]. However, even at this highly aciduric stage, the mineral balance and composition of the microflora could possibly be reversed by modification of the acidic environment, e.g. as a result of sugar restriction [De Stoppelaar et al., 1970].

The current state of caries risk assessment provides weak evidence to predict high-risk individuals. There is limited success to predict high-risk individuals and explain variations in caries among individuals, population subgroups or between populations [Aleksejūniene et al., 2009].

Wendt et al. [2001] evaluated a new strategy for the dental care of preschool children considering an early caries risk assessment and prevention in pre-school children. Children were studied from 1 to 6 years of age (intervention group) and a group of children from the same clinic (historical control) was used as a comparison group. On the basis of the clinical examinations of the children and the interviews with the parents considering past caries experience, visible plaque, gingivitis, oral hygiene habits, use of fluoride, language, preventive behaviors, and medical factors (medications, chronic diseases) when the children were 3 years of age, the children in the intervention group were divided into four different risk groups (no risk, low risk, moderate risk, and high caries risk). At 6 years of age $81 \%$ in the intervention group were free of manifest carious lesions, compared with $55 \%$ in the comparison group $(p<0.001)$. The authors concluded that early primary prevention (before the onset of caries attack) and a structured and systematic approach to dental care for preschool children result in good oral health for the children and may be economically profitable for a society with organized public dental service for preschool children.

According to Hausen et al. [2007], a preventive regimen that includes multiple measures such as oral hygiene and dietary counseling coupled with noninvasive measures for controlling dental caries can significantly reduce caries increment among caries-active children living in an area where the overall level of caries experience is low. However, a further challenge would be to design a regimen that is not only efficacious but also cost-effective.

In summary, dental caries is a complex disease that may be studied through systems modeling. The current research base on caries risk assessment is weak, but provides some indications for caries prediction. There is a need for population-based comparative effectiveness research studies that evaluate social, biological, behavioral, and upstream policy and economic environments that impact on caries using nonlinear statistical models.

\section{Strategies for Determining Population-Based Risk Assessment for Caries (Presenter R. Watt)}

Medical thinking has been largely concerned with responding to the needs of sick individuals as part of a symptomatic strategy [Rose, 1992]. Two basic types of preventive approaches were previously described by Rose 
[1992]: the high-risk and the population approaches. The high-risk approach focuses attention on individuals at high risk who have been identified through screening tests [Rose, 1992]. Once identified, the high-risk individuals at the tail end of the disease distribution are then offered preventive support in an attempt to modify the course of the condition [Watt, 2005]. The essential tenet of Rose's [1992] work is that while strategies that focus on high-risk individuals may help these people reduce their risk of chronic disease, the impact on the total burden of disease at the population level may be disappointing. This is because numerous cases of risk-factor-related health problems may arise among the many people who are in the middle of the risk distribution. In contrast, by lowering the risk across the whole population, the number of attributable cases of disease are significantly reduced [Brown et al., 2007]. The vast majority of chronic disease risk factors uncovered during the past half century are associated with very modest increases in disease risk [Rockhill et al., 2000].

The high-risk approach has recognized limitations from a public health perspective such as prevention becomes medicalized: success is only palliative and temporary, strategy is behaviorally inadequate, it is a poor predictive value of screening tools, there are problems of feasibility and costs, the majority of new cases not restricted to the high-risk group, and it is an apolitical approach in nature which does not address the causes of the disease [Batchelor and Sheiham, 2002, 2006].

Rose [1992] sought to change the paradigm of risk factor modification by treating the whole population rather than high-risk individuals exclusively. According to Rose [1992], a large number of people at small risk may give rise to more cases than a small number of people at high risk. The high-risk approach to primary prevention has overshadowed the more important population approach [Beaglehole and Bonita, 1998]. In the population approach, public health measures are implemented to reduce the level of risk in the whole population, shifting the whole distribution to the left [Rose, 1992]. This more radical approach aims to address the underlying causes of disease across the whole population [Watt, 2005].

In the prevention of oral diseases, the high-risk approach has been largely dominant. It is now increasingly acknowledged that a combination of the high-risk and directed population approaches is the best option [Watt, 2005]. High-risk and population strategies are complementary. These estimates of the benefits of each may be useful to health planners, when combined with their local knowledge [Cooney et al., 2009].

Summary of the IADR Cariology

Research Group Symposium
Little improvement has taken place in the way caries prediction studies are reported. The multifactorial etiology of dental caries makes it likely that even the most sophisticated risk models will be of limited value in predicting future caries development very accurately. It must also be remembered that even a perfect marker is only capable of predicting a person's future caries experience if the conditions on which the prediction is based remain stable [Hausen, 1997].

The use of past caries experience as an indicator of future caries increment has justly been criticized by the argument that we should aim at identifying the high caries risk-susceptible individuals before there are any signs of past caries experience. The fact is, however, that past caries experience still remains the most powerful single predictor of future caries increment, and one could also argue that if some past caries experience is visible, it would be a mistake not to use this information in assessing risk for new caries [Hausen, 1997].

Kay and Locker [1998] examined the evidence of effectiveness of oral health promotion on caries, oral hygiene, oral health-related knowledge, and attitudes and behaviors. The authors systematically reviewed oral health promotion research evidence using electronic searching, iterative hand-searching, critical appraisal and data synthesis. They concluded that oral health promotion is effective for reducing caries, however, mass media programs have not been shown to be effective.

The dominant oral health preventive model in dentistry has evolved from the biomedical nature of dentistry, and an individual 'risk factor' focus of much of clinical oral epidemiology. It is increasingly recognized that this approach alone has failed to effectively reduce oral health inequalities, and may indeed have increased the oral health equity gap [Watt, 2007].

The skewed distribution of caries in the high-income countries has led to the emergence of targeted prevention programs toward those considered to be at high risk. Identifying and then targeting individuals at high risk is not a recommended strategy in public health because the risk assessment models are far from precise at individual levels, and there are practical problems in treating the identified individuals successfully [Burt, 2005].

The essential determinants of the health of society are thus to be found in its mass characteristics: the deviant minority can only be understood when seen in its societal context, and effective prevention requires changes that involve the population as a whole [Rose, 1992].

Based upon a biomedical model of disease, oral health professionals have traditionally focused preventive and 
educational action on altering those behaviors that were known to impact dental caries (microbiology; oral hygiene, sugar consumption, exposure to fluoride and use of dental services). The underlying theory behind this approach is that once individuals acquire the relevant knowledge and skills, they will alter their behavior to maintain good oral health [Watt, 2005]. A 'more of the same approach' is no longer an option. A paradigm shift is therefore needed away from the biomedical and behavioral approach to one that addresses the underlying social determinants of oral health through a combination of complementary public health strategies [Watt, 2007].

Fisher-Owens et al. [2007] presented a multilevel conceptual model, with the individual, family, and community levels of influence on oral health outcomes. The model incorporates the five key domains of determinants of health as identified in the population health literature: genetic and biological factors, the social environment, the physical environment, health behaviors, and dental and medical care. The model recognizes the presence of a complex interplay of causal factors and also incorporates the aspect of time, recognizing the progress of oral health diseases and influences on the child-host over time.

Attempts to differentiate levels of causation for health have been made. Again overlooking genetic influences, social and behavioral scientists and epidemiologists have shown how these physiological risk factors are in turn the result of individual risky behaviors. Behavioral scientists devise and test interventions to stop these risky behaviors (secondary prevention) or prevent their onset (primary prevention). The unit of intervention is usually an individual at risk, or, increasingly, a community-based approach is taken to reduce individual levels of risk. There is some debate as to whether community-based approaches, as currently conducted, are conceptually different from or simply another approach to individual-level changes [McKinlay and Marceau, 2000].

The primary determinants of disease are mainly economic and social, and therefore its remedies must also be economic and social. Medicine and politics cannot and should not be kept apart [Rose, 1992]. In conclusion, dental caries remains a significant public health problem and approaching high-risk groups alone will not address this disease. A combination of high-risk and population preventive approaches is required and there is a need for more radical preventive agenda.

\section{Reasons why the Genetic Background Is Important for Individual Caries Risk (Presenter A.R. Vieira)}

Although there is growing evidence that genetics contributes to caries susceptibility, historically there has been a lack of studies approaching the problem from this front. Caries is the consequence of the interaction of host factors, microbial infection, and substrate that favors the oral biofilm. It is not difficult to propose underlying genetic mechanisms modulating each of these factors, such as saliva factors, which influence bacterial adhesion or buffer capacity.

When looking at models that can incorporate genetic influences, it is important to consider the facts. In regards to types of diet and sugar consumption, a decline in caries experience based on DMFT/dmft scores in the past 5 decades in the US can be seen despite the very high sugar intake of the population. This decline is likely due to measures such as school-based education programs and fluoride exposure and in general promoting dietary changes alone have little or no impact in the future caries experience of the population. Water fluoridation has also shown an effect in caries experience in the US in the same period, with a decline in DMFT scores. However, not all individuals living in fluoridated areas experienced less disease, suggesting fluoride alone is not enough to protect everyone.

Human models, including studies with twins, provide evidence that caries has a genetic component. The classic Vipeholm study [Gustafsson et al., 1954] clearly showed that the increased exposure to foods rich in sugar increased the severity of caries, but even though the individuals in the study consumed caramel 4 four times a day in between meals, $20 \%$ of them had not developed any caries lesions after 1 year. This result suggests individual susceptibility also modulates caries experience. Only one publication related to the Vipeholm study looked at familial aggregation related to caries, and it showed that parents and siblings of subjects of the Vipeholm study who did not develop any caries lesions had significantly lower caries prevalence than the parents and siblings of the remaining subjects [Böök and Grahnén, 1953]. Twin studies also provide evidence that genetics influence caries. Several variables related to caries experience (i.e. number of teeth present, percentage of teeth restored, percentage of surfaces restored, percentage of teeth affected by caries, percentage of surfaces affected by caries) show statistically significant concordance rates in monozygotic twins, but not in dizygotic twins [Boraas et al., 1988]. These studies measure the heritability, or the 
amount of variation in the disease frequency that is due to genetic variation, and twins have been studied in the US, Brazil, and China, with heritability values ranging from 25 to $80 \%$, depending on the disease-related variable studied.

Animal models have also been used to investigate the genetic contribution to caries, particularly in Japan. The basic design of this approach involves crossing strains particularly susceptible to the disease with resistant strains. Loci in chromosomes 1, 2, 7, 8, and 17 were suggested to contribute to caries susceptibility [Suzuki et al., 1998; Uematsu et al., 2003; Nariyama et al., 2004].

Our studies of the genetic contribution to caries involve two study designs: candidate gene approaches and genome-wide scan approaches. Since caries is particularly influenced by the environment (oral hygiene habits, types of diet, fluoride exposure, access to care), the main emphasis of our group is on characterizing the study population to decrease heterogeneity. We observed that a collection of about 100 dry skulls derived from individuals that lived in the northeast of the United States more than 100 years ago had high caries experience, but not all specimens had signs of the disease [Rose and Vieira, 2008]. These individuals lived in times prior to organized dentistry and likely had the same oral hygiene practices and dietary habits as the general population. Since we have access to population samples from many areas, we decided to focus on groups with limited access to dental care and information about oral health. We performed the first genome-wide linkage studies in caries in a group of families from the Philippines. These families lived in rural areas and had similar cultural and behavioral habits. We detected suggestive linkage between low caries experience and loci 5q13.3, 14q11.2, and Xq27.1. Also, high caries experience was linked to loci 13q31.1 and 14q24.3 [Vieira et al., 2008]. The candidate gene approaches include three main groups of genes: genes involved in enamel development, saliva formation and composition, and immune response. These studies include populations from Tiquisate (Guatemala), Istanbul (Turkey), Pittsburgh (USA), and the Patagonian region of Argentina. All these populations have similar socioeconomic status and access to dental care. Initial results are very encouraging and suggest that associations can be found between these candidate genes and high caries susceptibility [Deeley et al., 2008; Patir et al., 2008; Ozturk et al., 2010].

In summary, we believe that genetic susceptibility to caries can be identified under specific experimental conditions. Several genes likely influence individual susceptibility to caries, and these include genes involved in enamel development, saliva function, and immune response.

\section{Acknowledgments}

The authors are grateful to the IADR Cariology, Diagnostic Sciences, and Oral Health research groups. Jaime A. Cury helped planning the symposium. Mary L. Marazita presented the last part of the symposium entitled 'Feasibility of Large Genetic Population-Based Studies on Caries'. Colgate provided financial support for the symposium. A.R. Vieira is supported by the NIH Grant R01-DE018914.

\section{References}

Aleksejūniene J, Holst D, Brukiene V: Dental Beaglehole R, Bonita R: Public health at the caries risk studies revisited: causal approaches needed for future inquiries. Int $\mathrm{J}$ Environ Res Public Health 2009;6:29923009.

American Dental Association: ADA Policy on Evicence-Based Dentistry. 2008. http:// www.ada.org/1754.aspx.

Batchelor P, Sheiham A: The limitations of a 'high-risk' approach for the prevention of dental caries. Community Dent Oral Epidemiol 2002;30:302-312.

Batchelor P, Sheiham A: The distribution of burden of dental caries in schoolchildren: a critique of the high-risk caries prevention strategy for populations. BMC Oral Health 2006; $6: 3$. crossroads: which way forward? Lancet 1998 351:590-592.

- Beck JD: Risk revisited. Community Dent Oral Epidemiol 1998;26:220-225

Böök JA, Grahnén H: Clinical and genetical studies of dental caries. II. Parents and sibs of adult highly resistant (caries-free) propositi. Odontol Revy 1953;4:1-53.

Boraas JC, Messer LB, Till MJ: A genetic contribution to dental caries, occlusion, and morphology as demonstrated by twins reared apart. J Dent Res 1988;67:1150-1155.

rown WJ, Hockey R, Dobson A: Rose revisited: a 'middle road' prevention strategy to reduce noncommunicable chronic disease risk. Bull World Health Organ 2007;85:886-887.
Burt BA: Concepts of risk in dental public health. Community Dent Oral Epidemiol 2005;33: 240-247.

- Cooney MT, Dudina A, Whincup P, Capewell S, Menotti A, Jousilahti P, Njølstad I, Oganov $\mathrm{R}$, Thomsen T, Tverdal A, Wedel H, Wilhelmsen L, Graham I; SCORE Investigators: Re-evaluating the Rose approach: comparative benefits of the population and high-risk preventive strategies. Eur J Cardiovasc Prev Rehabil 2009;16:541-549.

Deeley K, Letra A, Rose EK, Brandon CA, Resick JM, Marazita ML, Vieira AR: Possible association of amelogenin to high caries experience in a Guatemalan-Mayan population. Caries Res 2008;42:8-13.

de Savigny D, Adam T: Systems Thinking for Health Systems Strengthening. Geneva, Alliance for Health Policy and Systems Research, World Health Organization, 2009. 
-De Stoppelaar JD, Van Houte J, Backer Dirks O: The effect of carbohydrate restriction on the presence of Streptococcus mutans, Streptococcus sanguis and iodophilic polysaccharide-producing bacteria in human dental plaque. Caries Res 1970;4:114-123.

-Fisher-Owens SA, Gansky SA, Platt LJ, Weintraub JA, Soobader MJ, Bramlett MD, Newacheck PW: Influences on children's oral health: a conceptual model. Pediatrics 2007; 120:e510-e520.

- Gustafsson BE, Quensel CE, Lanke LS, Lundqvist $\mathrm{C}$, Grahnén H, Bonow BE, Krasse B: The Vipeholm Dental Caries Study. The effects of different levels of carbohydrate intake in 436 individuals observed for five years. Acta Odontol Scand 1954;11:232-364.

-Hausen H: Caries prediction - state of the art. Community Dent Oral Epidemiol 1997;25: $87-96$.

- Hausen H, Seppa L, Poutanen R, Niinimaa A, Lahti S, Kärkkäinen S, Pietilä I: Noninvasive control of dental caries in children with active initial lesions: a randomized clinical trial. Caries Res 2007;41:384-391.

Ismail AI, Bader JD; ADA Council on Scientific Affairs and Division of Science; Journal of the American Dental Association: Evidencebased dentistry in clinical practice. J Am Dent Assoc 2004;135:78-83.

Kao RT: The challenges of transferring evidencebased dentistry into practice. J Evid Based Dent Pract 2006;6:125-128.
Kay E, Locker D: A systematic review of the effectiveness of health promotion aimed at improving oral health. Community Dent Health 1998;15:132-144.

McKinlay JB, Marceau LD: To boldly go. Am J Public Health 2000;90:25-33.

Nariyama M, Shimizu K, Uematsu T, Maeda T: Identification of chromosomes associated with dental caries susceptibility using quantitative trait locus analysis in mice. Caries Res 2004;38:79-84.

Ozturk A, Famili P, Vieira AR: The antimicrobial peptide $D E F B 1$ is associated with caries. J Dent Res 2010;89:631-636.

- Patir A, Seymen F, Yildirim M, Deeley K, Cooper ME, Marazita ML, Vieira AR: Enamel formation genes are associated with high caries experience in Turkish children. Caries Res 2008;42:394-400.

Pitts NB: Modern concepts of caries measurement. J Dent Res 2004;83:C43-C47.

Rockhill B, Kawachi I, Colditz GA: Individual risk prediction and population-wide disease prevention. Epidemiol Rev 2000;22:176-180.

Rose EK, Vieira AR: Caries and periodontal disease: insights from two US populations living a century apart. Oral Health Prev Dent 2008;6:23-28.

Rose G: The Strategy of Preventive Medicine. New York, Oxford University Press, 1992.

Schwaninger M: The role of system dynamics within the systems movement; in Barlas Y (ed): Theme System Dynamics, in Encyclopedia of Life Support Systems (EOLSS), UNESCO, chap 6.63.2.4. Oxford, EOLSS Publishers, 2003/4.
Selwitz RH, Ismail AI, Pitts NB: Dental caries. Lancet 2007;369:51-59.

-Suzuki N, Kurihara Y, Kurihara Y: Dental caries susceptibility in mice is closely linked to the H-2 region on chromosome 17. Caries Res 1998;32:262-265.

Takahashi N, Nyvad B: Caries ecology revisited: microbial dynamics and the caries process. Caries Res 2008;42:409-418.

Uematsu T, Nariyama M, Shimizu K, Maeda T: Mapping of affected gene(s) to dental caries susceptibility on mouse chromosome 2 . Pediatr Dent J 2003;13:75-81.

-Vieira AR, Marazita ML, McHenry TG: Genome-wide scan finds suggestive caries loci. J Dent Res 2008;87:435-439.

Von Bertalanffy L: General System Theory: Foundations, Development, Applications. New York, Brazilier, 1968.

Watt RG: Strategies and approaches in oral disease prevention and health promotion. Bull World Health Organ 2005;83:711-718.

-Watt RG: From victim blaming to upstream action: tackling the social determinants of oral health inequalities. Community Dent Oral Epidemiol 2007;35:1-11.

Wendt LK, Carlsson E, Hallonsten AL, Birkhed D: Early dental caries risk assessment and prevention in pre-school children: evaluation of a new strategy for dental care in a field study. Acta Odontol Scand 2001;59:261-266. 